\title{
Evaluation of a Speculative Multithreading Compiler by Characterizing Program Dependences
}

\author{
Anasua Bhowmik \\ Department of Computer Science \\ and Automation \\ Indian Institute of Science \\ Bangalore, India \\ anasta@csa.iisc.ernet.in
}

\author{
Manoj Franklin \\ Electrical and Computer Engineering \\ Department and UMIACS \\ University of Maryland \\ College Park, MD 20742 \\ manoj@eng.umd.edu
}

\begin{abstract}
Speculative multithreading (SpMT) promises to be an effective mechanism for parallelizing non-numeric programs. Proper thread formation is crucial for obtaining good speedup in an SpMT system. We have developed an SpMT compiler framework for partitioning sequential programs into multiple threads. Since control and data speculations are the essence of SpMT execution model, inter-thread data dependences and inter-thread control predictions at run-time ploy crucial roles in affecting the performance of the SpMT system. Therefore, to evaluate existing SpMT compiler or hardware systems, and to design more effcient systems it is necessary to characterize the $d y$ namic program dependences carefully. In this paper, we have studied the run-time behaviors of inter-thread data and control dependences of the threads generated by our compiler in detail and used that for analyzing the performance. The analyses reveal that our compiler has successfully modeled the inter-thread data and control dependences of non-numeric applications and minimized them while generating the threads.
\end{abstract}

\section{Introduction}

Speculative multithreading (SpMT) is emerging as an effective mechanism for exploiting thread-level parallelism (TLP) from non-numeric programs. SpMT processors allow multiple threads to execute in parallel in the presence of ambiguous control and data dependences, and recover upon the detection of dependence violations.

Control and data speculations are the essence of
SpMT execution models. Both inter-thread data dependences and inter-thread control predictions at runtime play crucial roles in affecting the performance of the SpMT system. Therefore, it is important that we carefully characterize the execution behaviors of threads in order to gain a better understanding of performance and to evaluate the effectiveness of the compiler and the hardware.

Most SpMT processors $[5,10,12,14]$ rely on the compiler to generate proper threads so as to exploit the parallelism available in the programs. The SpMT compiler should try to partition the program such that the inter-thread data dependences are minimized during execution. The threads are spawned and executed speculatively and inter-thread control missspeculation leads to squashing of the threads. Therefore, the compiler should try to expose more predictable branches at the thread boundaries while hiding the less predictable branches inside the threads.

We have developed an SpMT compiler [2] to partition sequential programs into threads for running on SpMT processors. Our work differs from earlier works on SpMT compilation $[11,15,17]$ in a number of ways. Most of the earlier works $[11,17]$ primarily target looplevel parallelism, whereas our compiler exploits parallelism from the non-loop regions as well.

In this paper we used a simulation-based environment to evaluate the performance of the non-numeric programs partitioned by our SpMT compiler. In order to have a better understanding of the SpMT system performance and evaluate the effectiveness of our compiler algorithms, it is extremely important to perform a detailed analysis of the run-time behaviors of the inter-thread data dependences and control predictions 
of the generated threads. Our detailed study shows that our compiler has modeled the inter-thread data and control dependences efficiently. It has been able to minimize both inter-thread data and control dependences effectively in most of the programs. Moreover, our study further reveals the difference in access patterns for inter-thread memory and register data and indicates further opportunity of improvement for the SpMT compiler and the hardware. Our characterization of data and control dependences of the multithreaded programs help in understanding program behaviors in SpMT execution model and indicates the ways to improve the systern further.

The rest of the paper is organized as follows. In Section 2, we review the concepts of speculative multithreading and discuss the important issues related to the performance of the SpMT system. We present an overview of our SpMT compiler framework in Section 3. The experimental methodologies and the evaluation are presented in Section 4. Finally we conclude in Section 5.

\section{Speculative multithreading}

The central idea behind SpMT is to execute multiple threads obtained from a (sequential) program in parallel. A number of SpMT models have been proposed, including multiscalar [5], superthreading [14], DMT [1], clustered speculative multithreaded processor $[10]$, and CMP $[8,11]$. Speculative multithreading enables parallelization of applications, despite any uncertainty about (control or data) dependences that may exist between the parallel threads. The hardware speculates on dependences, and recovers whenever a speculation is found to be incorrect. This allows the SpMT compiler to do optimistic speculation, thereby improving the performance. Below we review some of the important aspects of SpMT.

\subsection{Importance of considering inter- thread data dependences}

Perhaps the most important factor affecting the SpMT program performance is inter-thread data dependences, which affect inter-thread data communication and determine how much TLP exists. In case of data dependences the consumer instruction has to delay the execution until the data is available from the producer. SpMT processors can employ data value prediction to overcome the delay caused by data dependences. However, in that case, data misprediction would lead to squashing and re-execution of the threads (or affected

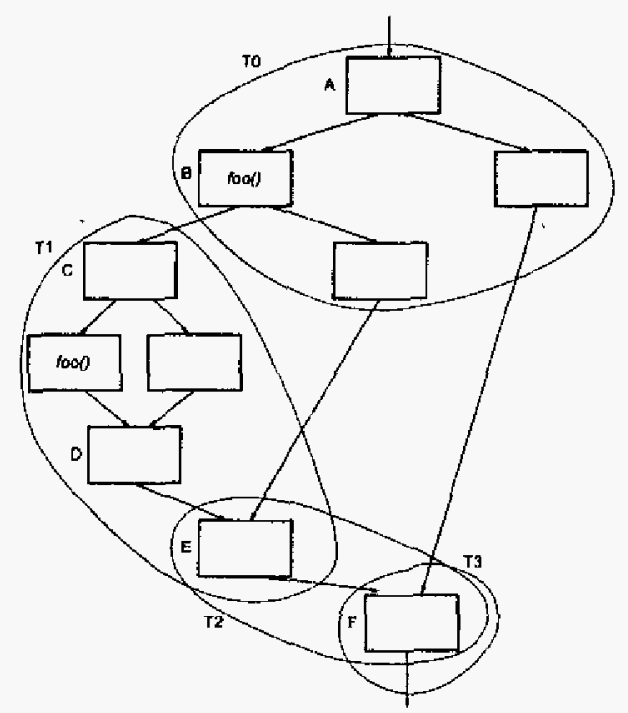

Figure 1: Structure of threads in an SpMT program

instructions). Moreover, unlike branch prediction, the accuracy of data value prediction is not very high.

Therefore, the compiler should try to partition the program in such a way that inter-thread data dependences be minimized. However, it is not possible to detect all data dependences at compile time because of aliasing. It is also not possible to accurately determine the relative timing of the dependent instructions in different threads because of factors like conditional branches, cache misses and dynamic instruction scheduling. The compiler can use profile information, heuristics and intra-thread scheduling to minimize inter-thread data dependences.

\subsection{Importance of considering inter- thread control dependences}

Inter-thread control dependences play a major role in the performance of the SpMT systems. The threads are generally spawned speculatively where the existence of the spawned thread is control dependent on a conditional branch after the spawning point and if that conditional branch is mis-predicted then the speculative thread has to be discarded. Therefore, the compiler should partition the program so that the conditional branches at thread boundaries have high prediction accuracies. The conditional branches with poor prediction accuracies can be encapsulated within the thread because an intra-thread branch misprediction does not directly affect the execution of a successor speculative thread whose existence depends on a different conditional branch. Below we classify 
the threads into three categories based on the control dependence relationship of the threads with their spawning points.

Loop-centric Threads: Each loop iteration can be specified as a thread that executes in parallel with other iterations. The only form of control dependences shared between multiple threads of this kind are loop termination branches. Therefore, loop-centric threads have very high inter-thread control prediction accuracies. However, in non-numeric programs, many of the loops do not have adequate parallelism. Moreover the programs tend to spend lot of time outside of the loops and so it is important to form threads from non-loop regions also.

Control Speculative Threads: Speculative spawning is the essence of SpMT. In Figure 1, thread T1 is speculative because it is spawned from block $A$ and the execution of $T 1$ is control dependent on the conditional branch between blocks $B$ and $C$. Speculative spawning is particularly desirable when the control flow is likely to take the speculated path more often than the other possible paths.

Control Non-speculative Threads: Many nonnumeric programs also tend to have a noticeable number of control mispredictions, necessitating frequent recovery actions. Therefore, it is also important to exploit control independence, possibly by identifying threads that are non-speculative from the control point of view. When executing such a non-speculative thread in parallel with its spawning thread, a branch misprediction within the initiator does not affect the non-speculative thread's existence, although it can potentially affect its execution (through inter-thread data dependences). Effective use of control independence information thus helps to reach distant code, despite the presence of mispredicted branches in between. In Figure 1, T3 is non-speculative, as it is spawned from $A$ and the execution of $T 2$ is controlindependent of the path taken to reach $T 3$ from $A^{1}$.

\subsection{Prior compiler work on SpMT}

Most of the SpMT proposals advocate thread formation at compile time, because the hardware is quite limited in its ability to analyze large sections of code. There have been several implementations of compilerbased thread generation for SpMT systems, including Hydra [6], superthreading [17], multiscalar [15], clustered speculative multithreaded processor [9], and Stampede [13]. Among these, [6], [13] and [17], focus

\footnotetext{
Notice that if a speculative thread To spawns a nonspeculative thread $T 3$, then $T 3$ is non-speculative from To's point of view, but may not be from TO's initiator's point of view.
}

mainly on loop-centric threads. [15] was the first major effort to partition the entire program, including the non-loop regions for parallel execution. [9] also partitions the entire program. It relies entirely on profiling to determine program partitions.

\subsection{Overview of our SpMT compiler framework}

In this section we give an overview of our SpMT compiler framework. The details of our compiler can be found in $[2,3]$. While partitioning a sequential program, our compiler considers data dependences, control dependences, and thread size together, to decide a good partitioning. It is built using the SUIF compiler framework [16], The control dependence in the program is captured by building the control flow graph of the procedures in the program. Profiling is done to determine the likely paths in the program. The compiler generates speculative threads residing within the likely paths. Also, the parallelism available at the control independent regions are exploited by starting threads from control-independent regions. The compiler tries to capture inter-thread data dependences as accurately as possible with the help of interprocedural data flow aralysis and inter-thread data dependence modeling.

\begin{tabular}{||l|l|}
\hline \multicolumn{1}{|c|}{ Configuration of a Processing Element(PE) } \\
\hline Instruction window & 64 instructions \\
\hline Issue width & up to 4 instructions/cycle \\
\hline Fetch width & up to 4 instructions/cycle \\
\hline Integer Units & 4 with I cycle latency \\
\hline Load-Store Units & 2 with 1 cycle latency \\
\hline Multiplier Unit & 1 with 12 cycles latency \\
\hline Branch Unit & 1 with 1 cycle latency \\
\hline Floating Point Unit & 2 with 2 cycles latency \\
\hline
\end{tabular}

\begin{tabular}{|c|c|}
\hline \multicolumn{2}{|l|}{ SpMT processor Parameters } \\
\hline Inter-PE communication latency & 2 cycles \\
\hline $\begin{array}{l}\text { Overhead of assigning } \\
\text { a thread to a PE }\end{array}$ & 2 cycles \\
\hline Thread pre-emption overhead & 2 cycles \\
\hline
\end{tabular}

Figure 2: SpMT processor parameters

\section{Experimental evaluation}

To evaluate the effectiveness of the threads generated by our compiler, we simulate the threaded programs in an SpMT simulator, modeled on top of a trace-driven simulator. Each processing element has its own program counter, fetch, decode and execution units. The 


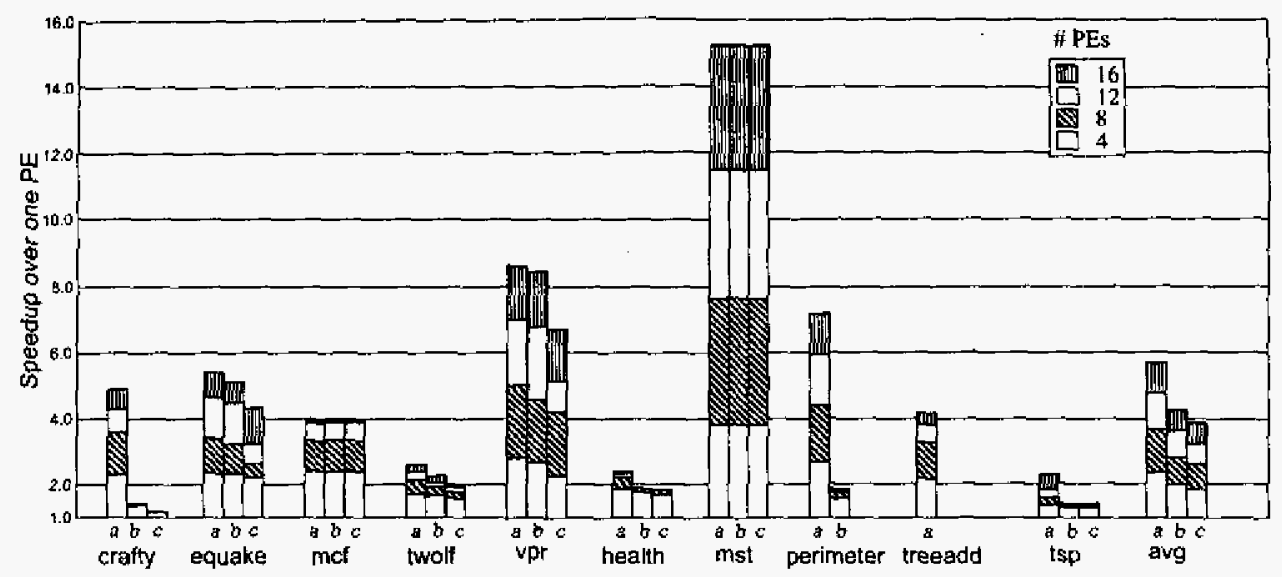

Figure 3: Speedup obtained with different types of threads. a: Speculative + Non-Speculative + Loop-centric threads; $b$ : Non-Speculative + Loop-centric threads; $c$ : Loop-centric threads only;

SpMT processor parameters used for our evaluation are shown in Figure 2. The simulator also models a shared 256 KB L1 d-cache with 1 cycle latency, and a miss latency of 10 cycles. Branch predictions are done using a 2-level Pap scheme. A hybrid data value predictor is used for predicting the results of instructions whose operands are unavailable at decode time. ${ }^{2}$

Benchmarks: For benchmarks, we use 10 programs: 5 from SPEC2000 and 5 from the Olden suite, all written in C. For the SPEC benchmarks, we "fast forward" the first 500 million instructions. For the Olden benchmarks there is no need to fast forward as they do not have significant initialization phases. We simulate all the programs except mef and perimeter for one billion instructions after fast forwarding (the parallelism values are found to be stable at one billion instructions); mef terminated after 700 million instructions while perimeter terminated after 500 million instructions. For the SPEC benchmark programs we use the train data set as profiling input, and the ref data set as simulation input (except for mcf). For mcf, we use the Igred input from the MinneSPEC [7] input set, as its ref input had very high memory requirements. In Olden benchmarks we use the same input sizes as [4] for the simulation and smaller size input for profiling.

\subsection{Evaluation of basic thread genera- tion scheme}

To evaluate the effectiveness of our basic partitioning algorithm, we measure the speedup (measured with respect to the sequential execution) obtained in the

\footnotetext{
${ }^{2}$ The library code is not parallelized, as we use the standard libraries in our experiments. The serial execution of the library code provides a conservative treatment to our parallelism values.
}

programs by increasing the number of PEs from 4 to 16. Figure 3 presents speedup obtained with 4,8 , 12 , and 16 PEs for three possible combinations of the different types of threads mentioned in Section 2.2 the loop-centric threads, speculative threads and nonspeculative threads.

From Figure 3 we see that beside loop-centric threads and non-speculative threads, we also need speculative threads to exploit the full performance potential of most of the programs. For example, crafty has good speedup only when all three types of threads are used. From Figure 3, we find that the compiler has been very successful in extracting parallelism from both non-loop programs (vpr, crafty, perimeter, treeadd) and loop-based programs (equake, mcf, mst). The fact that these benchmarks show good speedup and scalability indicates that the compiler has been successful in extracting parallelism from all parts of these programs.

The speedups are not quite good for twolf, heal th, and tsp. The programs twolf and health spend most of their time in loops that have very little parallelism. The poor parallelism in tsp is due to long latency multiplications that give rise to lots of unresolved interthread data dependences. In the next two subsections, we analyze the results shown in the bars a of Figure 3 (i.e., for all threads) further by looking at the dynamic control and data dependence behaviors of the threads in details.

\subsection{Characterization of inter-thread control prediction}

As discussed in Section 2.2, the compiler should try to minimize the inter-thread branch mispredic- 


\begin{tabular}{|c|c|c|c|c|c|}
\hline \multirow{2}{*}{$\begin{array}{l}\text { Prog. } \\
\text { Name }\end{array}$} & \multirow{2}{*}{$\begin{array}{c}\text { Overall } \\
\text { Branch Pred. } \\
\text { Accuracies }\end{array}$} & \multicolumn{2}{|c|}{ Inter-thread Branches } & \multicolumn{2}{|c|}{ Intra-thread Branches } \\
\hline & & \% Branches & $\begin{array}{l}\text { Prediction } \\
\text { Accuracies }\end{array}$ & \% Branches & $\begin{array}{l}\text { Prediction } \\
\text { Accuracies }\end{array}$ \\
\hline crafty & $92.41 \%$ & $7.8 \%$ & $94.36 \%$ & $92.2 \%$ & $92.25 \%$ \\
\hline equake & $93.42 \%$ & $61.4 \%$ & $99.51 \%$ & $38.6 \%$ & $83.72 \%$ \\
\hline Incf & $97.80 \%$ & $21.4 \%$ & $99.90 \%$ & $78.6 \%$ & $97.23 \%$ \\
\hline twolf & $88.19 \%$ & $29.3 \%$ & $98.41 \%$ & $70 . \overline{7 \%}$ & $83.95 \%$ \\
\hline vpr & $99.32 \%$ & $9.1 \%$ & $100.00 \%$ & $90.9 \%$ & $99.25 \%$ \\
\hline health & $99.75 \%$ & $70.8 \%$ & $99.98 \%$ & $29.2 \%$ & $99.18 \%$ \\
\hline mst & $91.35 \%$ & $8.4 \%$ & $100.00 \%$ & $91.6 \%$ & $90.56 \%$ \\
\hline perimeter & $8 \overline{8.80 \%}$ & $2.8 \%$ & $79.54 \%$ & $97 . \overline{2 \%}$ & $89.06 \%$ \\
\hline treeadd & $95.28 \%$ & $0.0 \%$ & $0.00 \%$ & $100.0 \%$ & $95.28 \%$ \\
\hline tsp & $95.20 \%$ & $32.0 \%$ & $98.61 \%$ & $68.0 \%$ & $93.60 \%$ \\
\hline
\end{tabular}

Table 1: Inter-thread and intra-thread branch prediction statistics

tions. Otherwise lots of squashing and re-execution of threads would take place. Therefore, it is important to see the efficiency of our compiler in reducing interthread control mispredictions. Our compiler tries to reduce inter-thread control mispredictions by spawning non-speculative threads from the control independent points and also by spawning speculative threads from the likely paths in the program.

In Table 1, we present the branch prediction statistics. Table 1 shows that except for perimeter, in all other benchmarks, inter-thread branch prediction accuracies are significantly higher than the intra-thread branch prediction accuracies and the overall branch prediction accuracies. This indicates the success of our compiler in exposing the more predictable branches at thread boundaries.

In Table 1, we see that equake, mcf, twolf, health, and tsp have significant proportions of interthread branches. In equake and health, the percentage of inter-thread branches are higher than that of intra-thread branches and also these branches have very high prediction accuracies. This is due to the fact that both these prograns spend more than $90 \%$ of the time in loop-centric threads and the interthread branches consist of mainly the loop terminating branches, resulting in high inter-thread branch prediction accuracies. The inter-thread branches in mcf, vpr, mst also mostly consist of loop-terminating branches.

In crafty, perimeter, and treeadd, some of the branches, on which the speculative threads are dependent, are included inside another thread. In treeadd and perimeter there are no loops and the program works by making recursive function calls. There are multiple function calls after a single conditional branch and speculative threads are spawned for every function call from before the conditional branch.
Therefore in this case there are successive speculative threads without any intervening conditional branch. From Figure 3, we see that in all three programs speculative threads achieve good speedup therefore the intra-thread branches, on which the speculations are made, are likely to have high accuracies as well.

In perimeter, the inter-thread branch prediction accuracies are lower than the intra-thread branch prediction accuracies. This is because of the overall poor branch prediction accuracy of the program. However the percentage of inter-thread branches is only $2.8 \%$ and as explained above not all branches, on which the speculations are based, are exposed at the thread boundaries.

\subsection{Characterization of inter-thread data dependences}

In this subsection we characterize the dynamic data dependence behaviors of the programs and correlate them with the program performance. Various statistics of inter-thread dynamic register and memory data dependences for 4 PEs are shown in Table 2.

For example, from Table 2 we see that, crafty has an average inter-thread register dependence of 11.54 . Out of this, 3.34 requests are made to inter-thread register whose values are not yet available. In crafty, out of an average of 3.34 , only an average of 0.56 unresolved register values are correctly predicted and the consumer instructions have to stall for the remaining 2.78 inter-thread register dependences.

From Table 2 we see that the programs that achieve high speedup do not have high inter-thread data dependences that cause stalls. For example, the average value for inter-thread register dependences cansing stalls for crafty is 2.78 and that for vpr is 2.92 . These values are not high, considering the average dy- 


\begin{tabular}{||l|r|r|r|r||r|r|r|r||}
\hline \multirow{2}{*}{$\begin{array}{l}\text { Program } \\
\text { Narae }\end{array}$} & \multicolumn{2}{|c|}{ A verage Inter-thread Register Dependences } & \multicolumn{3}{|c|}{ Average Inter-thread Memory Dependences } \\
\cline { 2 - 9 } & Total & Unresolved & $\begin{array}{r}\text { Correct } \\
\text { value }\end{array}$ & $\begin{array}{r}\text { Causing } \\
\text { Stalls }\end{array}$ & Total & Unresolved & $\begin{array}{r}\text { Correct } \\
\text { value }\end{array}$ & $\begin{array}{r}\text { Causing } \\
\text { Stalls }\end{array}$ \\
\hline \hline crafty & 11.54 & 3.34 & 0.56 & 2.78 & 8.14 & 1.55 & 0.53 & 1.02 \\
\hline equake & 15.48 & 4.99 & 1.12 & 3.87 & 2.26 & 0.52 & 0.01 & 0.51 \\
\hline mcf & 2.07 & 0.37 & 0.30 & 0.07 & $\overline{3.85}$ & 0.07 & 0.04 & 0.03 \\
\hline twolf & 5.74 & 1.97 & 0.60 & 1.37 & 5.23 & 0.80 & 0.26 & 0.54 \\
\hline vpr & 16.37 & 4.09 & 1.17 & 2.92 & 4.90 & 1.78 & 1.46 & 0.32 \\
\hline health & 3.04 & 1.99 & 0.00 & 1.99 & 2.10 & 0.01 & 0.00 & 0.01 \\
\hline mst & 4.58 & 0.07 & 0.07 & 0.00 & 2.71 & 0.13 & 0.05 & 0.08 \\
\hline perimeter & 8.16 & 2.17 & 0.40 & 1.77 & 5.71 & 1.20 & 0.68 & 0.52 \\
\hline treeadd & 11.00 & 4.10 & 1.13 & 2.97 & 8.55 & 3.37 & 2.56 & 0.81 \\
\hline tsp & 66.43 & 28.14 & 0.41 & 27.73 & 8.77 & 1.16 & 1.14 & 0.02 \\
\hline \hline
\end{tabular}

Table 2: Inter-thread average dynamic register and memory dependence statistics for 4 PEs

namic thread sizes of crafty and vpr are 93.5 and 80.1 , respectively. Similarly, mst, which has the highest parallelism, does not have any inter-thread register dependences that can cause stalls. On the other hand, in health the average inter-thread register dependences that cause stajl has value 1.99. This is a significant dependence, considering that the dynamic thread size of health is only 8.9 and this is also evident from the small speedup of health in Figure 3. tsp also has very high unresolved register dependences, resulting in small speedup. In tsp the very high unresolved register dependences are due to long latency multiplication and floating point operations.

From Table 2 we find that most programs have a higher number of register dependences causing stalls than that of memory dependences. However, even a small amount of memory dependences can cause more stalls if they result in cache misses. For example, in mcf, although the inter-thread dependences are not high, the cache miss rates are very high for both the intra-thread and the inter-thread memory dependences. Also, the data value prediction accuracy is higher for memory data than for register data. This is because the programs often load the same data from memory and this is easier to predict than the register values.

While building an inter-thread data dependence model the compiler tries to limit the number of unresolved inter-thread data dependences ${ }^{3}$ within 10 and adding up the unresolved register and memory data dependences, we can see that the value does not exceed 10 except for tsp. This validates our data dependence modeling.

In order to evaluate the inter-thread register dependence patterns, we measure the span of the inter-

\footnotetext{
${ }^{3}$ Data Dependence Count Model [2]
}

thread register dependences in Figure 4, where distances are measured in terms of dynamic threads. The $\mathrm{X}$-axis denotes the distances in dynamic threads and the $\mathrm{Y}$-axis denotes the fraction of register dependences that lie within specific dynamic thread distance. The graphs in (a) and (b) show the measurement for resolved and unresolved inter-thread register dependences for $4 \mathrm{PEs}$, respectively. Similarly, the span of inter-thread memory dependences are shown in Figure 5.

In Figure 4(a), we see that for mcf, health, mst and tsp the resolved register dependences are corning either from the immediate predecessor or from the threads that are more than ten dynamic threads apart. All the above programs are loop-centric, and for such programs most of the resolved register dependences are loop carried dependences coming from the last iteration. For all other benchmarks, the resolved register dependences are spanned more or less uniformly among the predecessor threads.

In Figure 4(b), we see that more than $50 \%$ of the unresolved register dependences are due to the immediate predecessor. In the loop based programs mcf, health, mst and tsp all the unresolved dependences are coming from the last iteration. Since our compiler and SpMT model support out-of-order thread spawning and execution [2], in a 4 PE SpMT processor there can be unresolved dependences due to threads that are more than 4 threads apart. However, except for twolf and perimeter, in all other programs nearly $100 \%$ of the unresolved dependences are due threads that are at most 4 threads away. This indicates that there is no stalls due to out-of-order execution.

In Figure 5(a), we see that in all benchmarks, except tsp, more than $50 \%$ of the resolved memory dependences are accessing threads that are more than 10 threads apart. Like register dependences, in case 


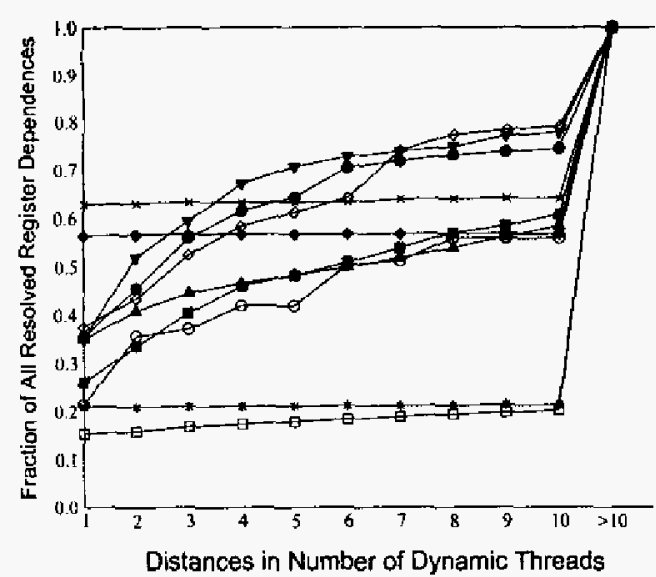

(a) $4 \mathrm{PEs}$

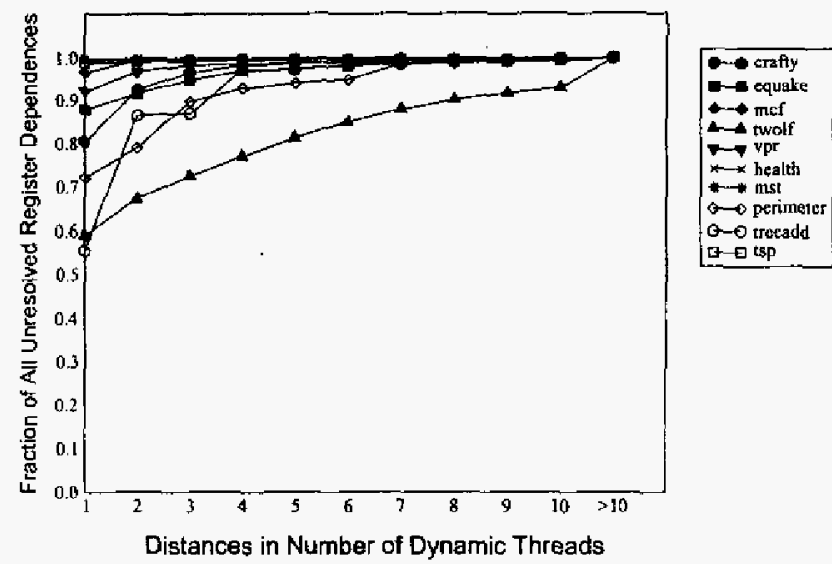

(b) $4 \mathrm{PEs}$

Figure 4: Distribution of register data dependences in terms of distances in dynamic threads

of memory dependences also, in the programs mcf, health, mst and tsp all the resolved dependences are coming from the previous iterations or from the threads that are far away.

In Figure 5(b), we see that the unresolved memory dependences span a larger distance than unresolved register dependences and indicates the existence of unresolved memory dependences due to out-of-order execution. Except for health, most of the unresolved memory dependences in other programs are mainly coming from nearby predecessors. Like unresolved register dependences, in mst and tsp all unresolved memory dependences are coming from immediate predecessor. Overall, the register and memory dependence patterns are quite different in other programs.

\section{Conclusions}

Speculative multithreading is emerging as an important parallelization method for non-numeric programs. Judicious partitioning of a sequential program into threads is necessary to exploit parallelism in SpMT processors. We have developed an SpMT compiler for partitioning sequential programs into multiple threads. The inter-thread data dependences and control dependences are extremely important in achieving good speedup. So the compiler should model the interthread data and control dependences as accurately as possible and generate the threads such that the dependences be minimized.

In this paper we used a simulation-based environment to evaluate the performance of the non-numeric programs partitioned by our SpMT compiler framework. We studied the inter-thread control and data dependences of the programs and analyzed the performances with the help of them. Our study shows that the compiler has modeled the inter-thread data and control dependences efficiently. It has been able to minimize both inter-thread data and control dependences effectively in most of the programs. However, lack of parallelism in case of long latency operations and the different access patterns of memory and register dependences indicates possible improvement for the compiler. Our characterization of data and control dependences of the multithreaded programs help in understanding program behaviors in SpMT execution model and indicates the ways to improve the system further.

\section{Acknowledgements}

This work was supported by the U.S. National Science Foundation (NSF) (through grants CCR 0073582 and CCR 9988256) and Intel Corporation.

\section{References}

[1] H. Akkary and M. A. Driscoll. "A Dynamic Multithreading Processor," in Proc. of Intl. Symp, on Microarchitecture (MICRO-\$1), 1998.

[2] A. Bhowmik and M. Franklin. "A General Compiler Framework for Speculative Multithreaded Processor," in IEEE Transactions on Parallel and Distributed Systems, Vol 15, Issue 8, August 2004.

[3] A. Bhowmik and M. Franklin. "A Fast Approximate Interprocedural Analysis for Speculative

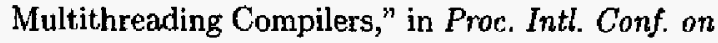
Supercomputing (ICS), 2003. 


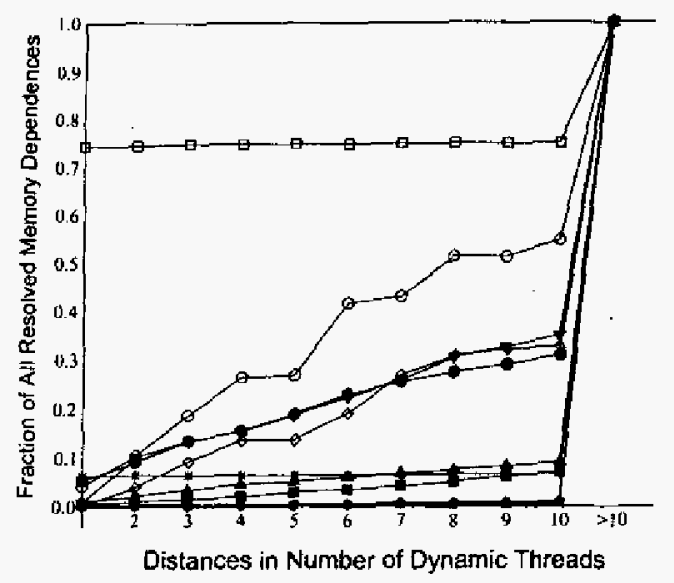

(a) 4 PEs

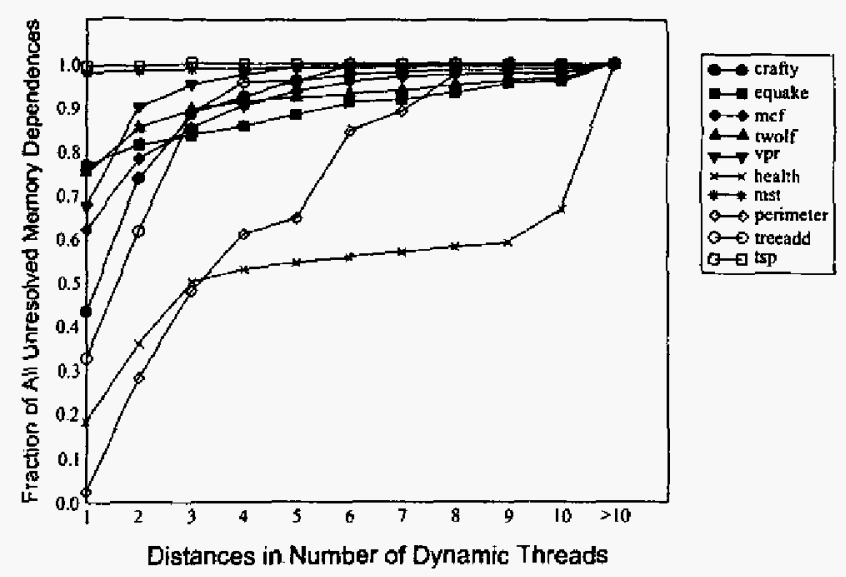

(b) 4 PEs

Figure 5: Distribution of memory data dependences in terms of distances in dynamic threads

[4] M. C. Carlisle and A. Rogers. "Software Caching and Computation Migration in Olden'," in Symp. on Principles and Practice of Parallel Programming, 1995.

[5] M. Franklin "Multiscalar Processors," Kluwer Academic Publishers, 2002.

[6] L. Hammond, M. Willey and K. Olukotun. "Data Speculation Support for a Chip Multiprocessor," in Proc. ACM Conf. on Anchitectural Support for Programming Languages and Operating Systems (ASPLOS), 1998.

[7] A. J. KleinOsowski and D. J. Lilja. "MinneSPEC: A New SPEC Benchmark Workload for Simulation-Based Computer Architecture Research," Comp. Arch. Letters, V 1, Jun 2002

[8] V. Krishnan and J. Torrellas. "A ChipMultiprocessor Architecture with Speculative Multithreading," in IEEE Transactions on Computers, Vol. 48, No. 9, September 1999.

[9] P. Marcuello and A. Gonzalez. "Thread Spawning Schemes for Speculative Multithreading," in Proc. Intl. Symp. on High-Performance Computer Architecture, 2002.

[10] P. Marcuello and A. Gonzalez. "Clustered Speculative Multithreaded Processors," in Proc. Intl. Conf. on Supercomputing (ICS), 1999.

[11] K. Olukotun, L. Hammond, and M. Willey. "Improving the Performance of Speculatively Parallel Applications on the Hydra CMP," in Proc. Intl. Conf. on Supercomputing (ICS), 1999.

[12] J. G. Steffan and T. Mowry. "The Potential for Using Thread-Level Data Speculation to Facilitate Automatic Parallelization," in Proc. of Intl. Symp. on High-Performance Computer Architecture (HPCA), 1998.
[13] J. G. Steffan and T. Mowry. A. Zhai, C. B. Colohan, J. G. Steffan and T. C. Mowry. "Compiler Optimization of Scalar Value Communication Between Speculative Threads," in Proc. Intl. Conf. on Architectural Support for Programming Languages and Operating Systems (ASPLOS-X), 2002.

[14] J-Y. Tsai and P-C. Yew. "The Superthreaded Architecture: Thread Pipelining with Run-Time Data Dependence Checking and Control Speculation," in Proc. Intl. Conf. on Parallel Architectures and Compilation Techniques (PACT), 1996.

[15] T. N. Vijaykumar and G. S. Sohi. "Task Selection for a Multiscalar Processor," in Proc. Intl. Symp. on Microarchitecture (MICRO-31), 1998.

[16] R. Wilson, R. French, C. Wilson, S. Amarsinghe, J. Anderson, S. Tjiang, S. Liao, C.-W. Tseng, M. Hall, M. Lam and J. Hennesy. "SUIF: An Infrastructure for Research on Parallelizing and $O p$ timizing Compiler," ACM SIGPLAN Notices, Vol. 29, No. 12, December 1996.

[17] B. Zheng, J.-Y. Tsai, B. Y. Zang, T. Chen, B. Huang, J. H. Li, Y. H.'Ding, J. Liang, Y. Zhen, P.-C. Yew, C. Q. Zhu. "Designing the Agassiz Compiler for Concurrent Multithreaded Architectures," in Workshop on Languages and Compilers for Parallel Computing ( $L C P C$ ), 1999. 